\title{
FEEDFORWARD CONTROL UNDER THE PRESENCE OF UNCERTAINTY
}

\author{
A. Faanes ${ }^{\dagger, *}$, S. Skogestad ${ }^{\dagger, * *}$ \\ ${ }^{\dagger}$ Department of Chemical Engineering, Norwegian University of Science and Technology, N-7491 Trondheim, Norway \\ * also affiliated with Statoil ASA, TEK, Process Control, 7005 Trondheim, Norway \\ ** Author to whom all correspondence should be addressed. E-mail: skoge@ chemeng.ntnu.no,
}

Tel. +47735941 54, Fax: +47 73594080

Keywords: Process control, Linear systems, Feedforward control, Uncertainty

\begin{abstract}
In this paper we study the effect of model errors on the performance of feedforward controllers. In accordance with the sensitivity function for feedback control, we define the feedforward sensitivities, $S_{\mathrm{ff}}$ (feedforward from disturbance) and $S_{\mathrm{ff}, \mathrm{r}}$ (feedforward from set-point), as measures for the reduction in the output error obtained by the feedforward control. For "ideal" feedforward controllers based on the inverted nominal model, the feedforward sensitivities equal the relative model errors, which must thus remain less than 1 for feedforward control to have positive (dampening) effect.

For some common model error classes we provide rules for when the feedforward controller is effective, and we also design $\mu$-optimal feedforward controllers.
\end{abstract}

\section{Introduction}

There is a fundamental difference between feedforward and feedback controllers with respect to their sensitivity to uncertainty. Feedforward control is sensitive to uncertainty in general (including steady-state), whereas feedback control is insensitive to uncertainty at frequencies within the system bandwidth. With no model error a feedforward controller may remove the effect of disturbances, but due to its dependence of the process model, it may actually amplify the effect of a disturbance if the model is wrong.

Most of the articles on feedforward control refer to industrial applications. Some control textbooks, e.g., [3, 16, 5, 13, 9, 4, $11,14]$, describe feedforward controllers and their design, and the advantages and disadvantages compared to feedback is discussed, qualitatively. A general quantitative frequency domain analysis of feedforward control under model uncertainty is proposed by [1] (and referred in [2]). The references [6, 9, 8, 14] give some typical numbers for the effect of model uncertainty on the output error.

In the context of IMC (Internal Model Control), Morari and Zafiriou [10] recommend a structure for the combined feedback-feedforward scheme that decouples the two functions. Scali and co-workers [7, 12], extend this work to de- rive an $\mathcal{H}_{2}$ optimal combination of feedback and feedforward controllers under the presence of uncertainty.

The aim of this article is to study feedforward control under the presence of uncertainty and answer the following basic questions: 1) How much does the feedforward controller reduce the control error?, 2) When is the feedforward controller amplifying the effect of disturbances on the outputs?, and 3) How can uncertainty be taken into account when the feedforward controller is designed?

\section{Feedforward control}

A block diagram where feedforward from a disturbance and the reference is combined with feedback, is shown in Figure 1. To analyze the effect of a given feedforward controller, we denote the feedback controller $K$ and the feedforward action from the disturbance $K_{\mathrm{ff}}$ and the reference $K_{\mathrm{ff}, r}$. With perfect measurements we then have (see Figure 1)

$$
u=\underbrace{K\left(y_{r}-y\right)}_{\text {Feedback }}+\underbrace{K_{\mathrm{ff}, r} y_{r}-K_{\mathrm{ff}} d}_{\text {Feedforward }}
$$

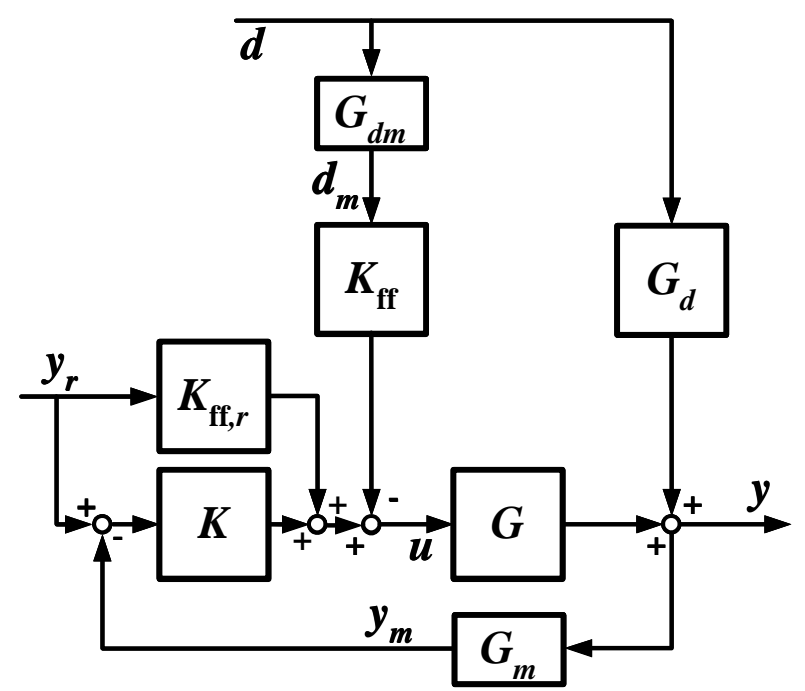

Figure 1: Block scheme for feedforward control combined with a feedback controller. We assume ideal measurements: $G_{d m}=$ 1 and $G_{m}=1$. 
The closed loop response for the combination of feedforward and feedback control is

$$
\begin{aligned}
e(s)= & y(s)-y_{r}(s) \\
= & S(s)\left(G_{d}(s)-G(s) K_{\mathrm{ff}}(s)\right) d(s) \\
& \quad-S(s)\left(I-G(s) K_{\mathrm{ff}, r}(s)\right) y_{r}(s)
\end{aligned}
$$

where $S(s)=(I+G(s) K(s))^{-1}$ is the feedback sensitivity function.

"Ideal" feedforward control An "ideal" feedforward controller based on inverting the nominal model (see e.g., [1, 2, $10]$ ), removes completely the effect of the disturbance and reference changes such that $e(s) \equiv 0$. We denote the "ideal" controller with an asterisk, and get from Equation (2)

$$
K_{\mathrm{ff}}^{*}=G^{-1} G_{d} ; \quad K_{\mathrm{ff}, r}^{*}=G^{-1}
$$

Designs of robustly optimized ( $\mu$-optimal) feedforward controllers presented later in this paper, confirm that this is a good controller as to use in some practical cases.

\section{Feedforward sensitivity functions}

The closed loop response for combined feedforward and feedback control in Equation (2) may be rewritten as follows

$$
e=S\left(S_{\mathrm{ff}} G_{d} d-S_{\mathrm{ff}, r} y_{r}\right)
$$

where we define the feedforward sensitivities as

$$
\begin{aligned}
S_{\mathrm{ff}} & =\left(I-G K_{\mathrm{ff}} G_{d}^{\dagger}\right) \\
S_{\mathrm{ff}, r} & =I-G K_{\mathrm{ff}, r}
\end{aligned}
$$

These express the effect of feedforward action on the control error. $G_{d}^{\dagger}$ denotes the generalized inverse of $G_{d}$. Feedback control is effective and improves performance as long as the gain of the sensitivity function $\|S\|<1$. Similarly feedforward control improves the performance if

$$
\left\|S_{\mathrm{ff}}\right\|<1 \text { and }\left\|S_{\mathrm{ff}, r}\right\|<1
$$

Here, an appropriate norm dependent on the definition of performance is used. It is due to these similarities that we denote $S_{\mathrm{ff}}$ and $S_{\mathrm{ff}, r}$ feedforward sensitivities. With no feedforward control $S_{\mathrm{ff}}=I$, and with "ideal" feedforward control $S_{\mathrm{ff}}=0$.

In the literature $S$ and $S_{\mathrm{ff}}$ are also denoted control ratio and feedforward control ratio, respectively [2]. More precisely, in [2], the feedforward control ratio is defined for single input single output (SISO) controllers as

$$
S_{\mathrm{ff}}=1-\frac{K_{\mathrm{ff}}}{K_{\mathrm{ff}}^{*}}
$$

where $K_{\mathrm{ff}}$ is the actual feedforward controller and $K_{\mathrm{ff}}^{*}$ is the "ideal" controller for the actual process. This is identical to the definition in Equation (5) for SISO controllers. Equation (5) is a reformulation of Equation 8, which extends to multivariable controllers, and in Equation 6 we have introduced the sensitivity function for feedforward from the reference.

In [2] a Nichols chart is used to determine requirements on the gain and phase error in $K_{\mathrm{ff}}$ relative to $K_{\mathrm{ff}}^{*}$ for a given disturbance dampening (e.g., 0.1) in $S_{\mathrm{ff}}$, since the Nichols chart has been convenient for the study of $h(j \omega)+1$ given a transfer function $h(j \omega)$. With tools like Matlab, it is now easy to study any transfer function by defining a finite number of frequencies and calculate the gain or phase shift over this set of frequencies. We follow this direct approach.

\section{The effect of model error with feedforward control}

In this section we restrict ourselves to single-input/singleoutput (SISO) processes, i.e., with one control input, $u$, one disturbance, $d$, and one output $y$. With a nominal process model, $y=G u+G_{d} d$, and an actual plant model $y^{\prime}=G^{\prime} u+G_{d}^{\prime} d$, the actual control error is:

$$
e^{\prime}=y^{\prime}-y_{r}=S^{\prime}\left(S_{\mathrm{ff}}^{\prime} G_{d}^{\prime} d-S_{\mathrm{ff}, r}^{\prime} y_{r}\right)
$$

where

$$
\begin{array}{ccc}
S^{\prime} & \stackrel{\text { def }}{=} & \frac{1}{1+G^{\prime} K} \\
S_{\mathrm{ff}}^{\prime} & \stackrel{\text { def }}{=} & 1-\frac{G^{\prime} K_{\mathrm{ff}}}{G_{d}^{\prime}} \\
S_{\mathrm{ff}, r}^{\prime} & \stackrel{\text { def }}{=} & 1-G^{\prime} K_{\mathrm{ff}, r}
\end{array}
$$

$S$ expresses the ratio between the output when a feedback controller is applied and when it is not (open loop). Similarly, $S_{\mathrm{ff}}^{\prime}$ and $S_{\mathrm{ff}, r}^{\prime}$ express the ratio of the output when feedforward is applied and the output when it is not. This follows by comparing the output error using control in Equation (9) with the output error when no control is applied $(u=0)$ :

$$
e^{\prime}=y^{\prime}-y_{r}=G_{d}^{\prime} d-y_{r}
$$

Note that for the case with no control $\left(K=0, K_{\mathrm{ff}}=0\right.$, $K_{\mathrm{ff}, r}=0$ ), we have $S^{\prime}=1, S_{\mathrm{ff}}^{\prime}=1, S_{\mathrm{ff}, r}^{\prime}=1$.

The actual sensitivity can be expressed in terms of the nominal sensitivity and the relative error as following

$$
S^{\prime}=S \frac{1}{1+E T}
$$

Here $S=(I+G K)^{-1}$ and $T=I-S$ are the nominal sensitivity and complementary sensitivity functions, respectively, and $E$ the relative error in $G$, i.e., $E=G^{\prime} / G-1$ (see also [15, Section 5.13]).

The "ideal" feedforward controller in Equation (3) gives with no model error

$$
S_{\mathrm{ff}}^{\prime *}=0, \quad S_{\mathrm{ff}, r}^{* *}=0
$$

With model error we get the result

$$
\begin{aligned}
S_{\mathrm{ff}}^{*} & =1-\frac{G^{\prime} / G_{d}^{\prime}}{G / G_{d}}=-E_{d} \\
S_{\mathrm{ff}, r}^{\prime *} & =1-\frac{G^{\prime}}{G}=-E
\end{aligned}
$$


Here, $E_{d}$ is the relative error in $G / G_{d}$ and $E$ the relative error in $G$. Thus for "ideal" controllers, $S_{\mathrm{ff}}^{\prime *}$ and $S_{\mathrm{ff}, r}^{\prime *}$ are equal to (except for the sign) the relative model errors in $G / G_{d}$ and $G$, respectively, and we have that the "ideal" feedforward action reduces the error as long as the relative modelling errors are less than one, i.e.,

$$
\begin{aligned}
\left|S_{\mathrm{ff}}^{\prime *}\right| & =\left|E_{d}\right|=\left|1-\frac{G^{\prime} / G_{d}^{\prime}}{G / G_{d}}\right|<1 \\
\left|S_{\mathrm{ff}, r}^{\prime *}\right| & =|E|=\left|1-\frac{G^{\prime}}{G}\right|<1
\end{aligned}
$$

If the model error (uncertainty) is sufficiently large, such that the relative error in $G / G_{d}$ is larger than 1 , then we see from Equation (16) that $\left|S_{\mathrm{ff}}^{\prime *}\right|$ is larger than 1 and "ideal" feedforward control makes control worse. This may quite easily happen in practice. For example, if the gain in $G$ is increased by $33 \%$ and the gain in $G_{d}$ is reduced by $33 \%$, then $S_{\mathrm{ff}}^{\prime *}=1-\frac{G^{\prime} / G}{G_{d}^{\prime} / G_{d}}=1-\frac{1.33}{0.67}=1-2=-1$. In words, the feedforward controller overcompensates for the disturbances, such that its negative counteractive effect is twice that of the original effect.

In Figure 2 we consider some examples of model uncertainties for "ideal" feedforward controllers, and use Equations (18) and (19) to determine when feedforward control should be used.

Example 1 In this example we consider feedforward control of the process illustrated in Figure 3. A hot flow with flow rate $q_{\text {in }}$ and temperature $T_{\text {in }}$ passes through tank 1 and into tank 2 where it is cooled by mixing with a cold flow with flow rate $q_{c}$ and temperature $T_{c} . T_{i n}$ is measured before the first tank. The outlet temperature, $T_{2}$, shall be kept constant despite temperature variations in the hot flow. To obtain this the measurement of $T_{\text {in }}$ is used by an "ideal" feedforward controller, $K_{\mathrm{ff}}=\left(\left(k_{d} / k\right) e^{-\theta s}\right) /\left(\tau_{1} s+1\right)$, to adjust $q_{c}$ to compensate for the variations.

The response to sinusoidal disturbances is shown in Figure 4.

\section{$\mu$-design}

We now consider combined gain and delay error in $G$, and define a $\mu$-optimalfeedforward controller as shown in Figure 5. We let the uncertainty weight, $W_{I}$, be diagonal with elements

$$
\begin{aligned}
& W_{I_{1}}=10^{-4} \\
& W_{I_{2}}=\frac{1.1 s+0.2}{0.5 s+1} \cdot \frac{(\xi)^{2} s^{2}+2 \cdot 0.838 \cdot \xi s+1}{(\xi)^{2} s^{2}+2 \cdot 0.685 \cdot \xi s+1}
\end{aligned}
$$

where $\xi=1 / 2.363$ and $W_{I_{1}}$ represents the uncertainty in $G_{d}$ (approximately zero) and $W_{I_{2}}$ represents the uncertainty in $G$ corresponding to $20 \%$ gain uncertainty and $\pm 1 \mathrm{~s}$ delay uncertainty [15, eq. (7.27)]. The performance weight, $W_{P}$, is chosen as a constant independent of frequency, and several values for $W_{P}$ is considered (from $10^{-4}$ to 1000). The $\mu$-controller is designed with $D$-K iterations using $\mu$-toolbox in Matlab (with scaling matrices of order 2). The delay difference between $G$ and $G_{d}$ is removed from the models used for the design, and the

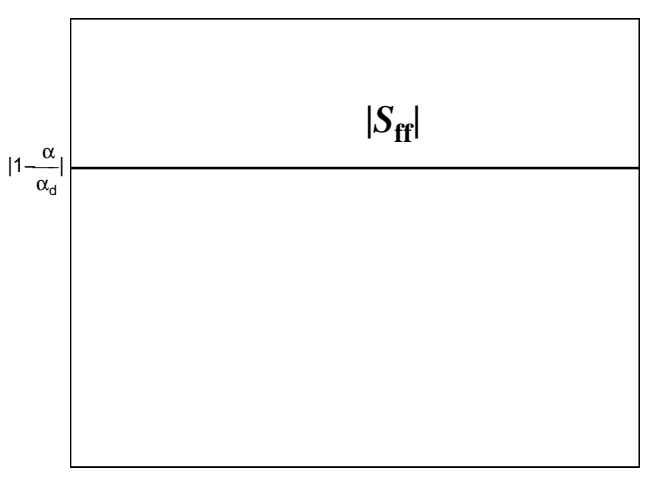

$\omega$

(a) Effect of gain uncertainty, $\left|S_{\mathrm{ff}}\right|=\left|1-\frac{\alpha}{\alpha_{d}}\right|$ with $G^{\prime}=\alpha G$ and $G_{d}^{\prime}=\alpha_{d} G_{d}$.

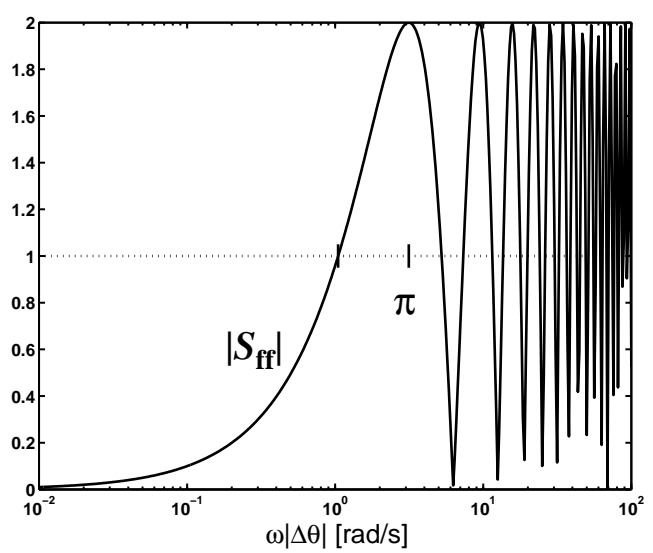

(b) Effect of time delay uncertainty, $\left|S_{\mathrm{ff}}\right|=$ $\left|1-e^{\Delta \theta j \omega}\right|$ where $\Delta \theta=\left(\theta_{d}^{\prime}-\theta^{\prime}\right)-\left(\theta_{d}-\theta\right)$, and $\theta_{d}^{\prime}, \theta^{\prime}, \theta_{d}$ and $\theta$ are the delays in $G_{d}^{\prime}, G^{\prime}, G_{d}$ and $G$, respectively. At low frequencies the effect is zero, but for high frequencies, it doubles the worst case error.

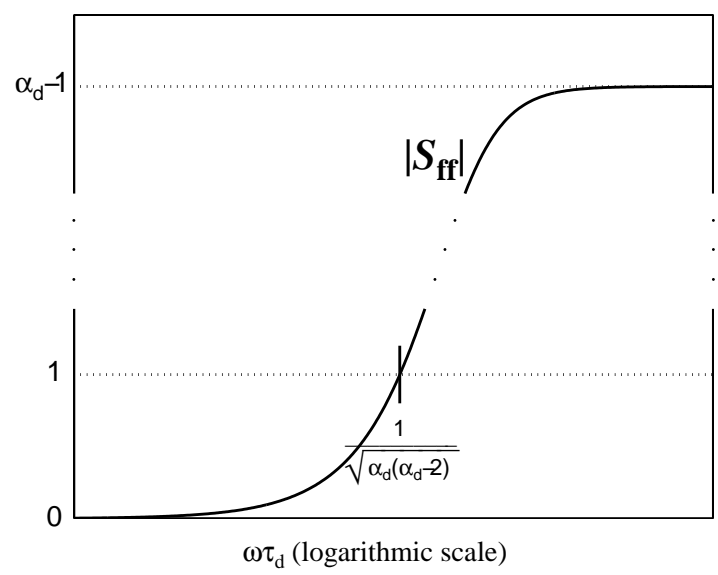

(c) Effect of time constant uncertainty, $\left|S_{\mathrm{ff}}\right|=$ $\left|1-\frac{\alpha_{d} \tau_{d} s+1}{\tau_{d} s+1}\right|$ with $G^{\prime}=G, G_{d}=G_{d_{0}} /\left(\tau_{d} s+1\right)$, $G_{d}^{\prime}=G_{d_{0}} /\left(\alpha_{d} \tau_{d} s+1\right)$. 


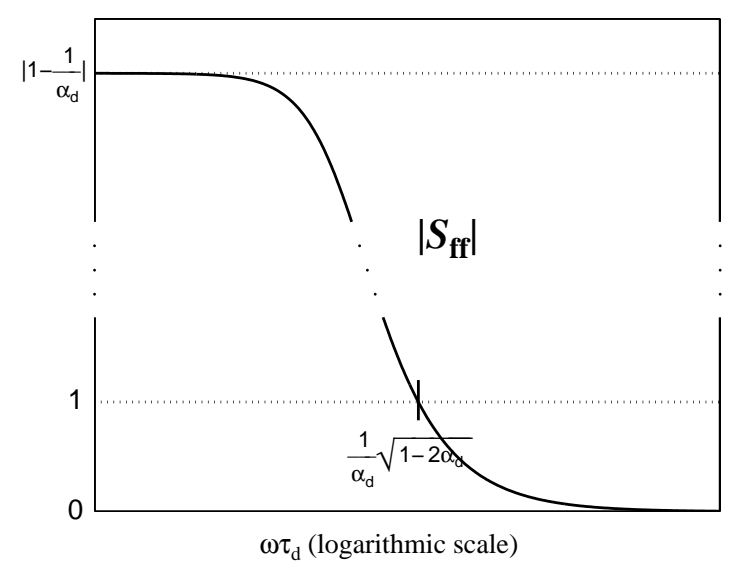

(d) Effect of uncertainty in the gain and the time constant keeping their ratio constant, $\left|S_{\mathrm{ff}}\right|=\left|\left(1-\frac{1}{\alpha_{d}}\right) \frac{1}{\tau_{d} s+1}\right|$ with $G^{\prime}=G, G_{d}=G_{d_{0}} /\left(\tau_{d} s+1\right), G_{d}^{\prime}=$ $\alpha_{d} G_{d_{0}} /\left(\alpha_{d} \tau_{d} s+1\right)$.

Figure 2: Effect of uncertainty on $S_{\mathrm{ff}}$ for SISO feedforward control

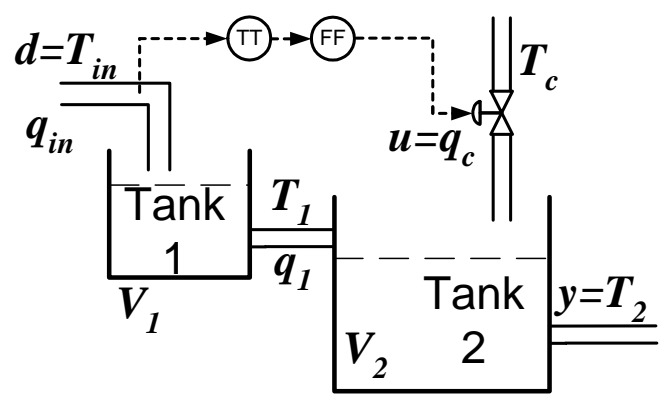

(a) Illustration of the process. Nominal data: $V_{1}^{0}=$ $100 \mathrm{l}, V_{2}^{0}=70 \mathrm{l}, q_{i n}^{0}=q_{1}^{0}=16 \mathrm{l} / \mathrm{s}, q_{c}^{0}=4 \mathrm{l} / \mathrm{s}$, $T_{c}^{0}-T_{2}^{0}=-40^{\circ} \mathrm{C}$

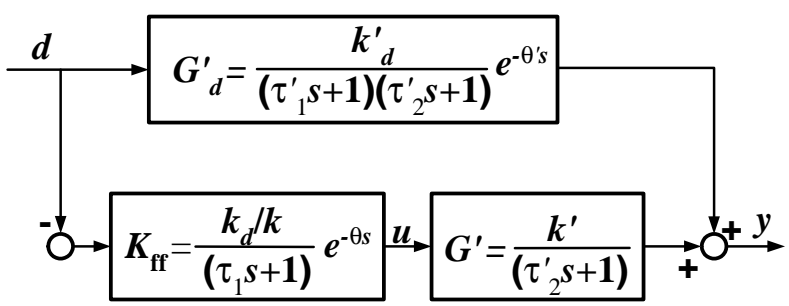

(b) Block diagram. Parameters derived from the nominal data: $k^{\prime}=-2, k_{d}^{\prime}=0.8, \tau_{1}^{\prime}=6.25, \tau_{2}^{\prime}=3.5$. In addition there is a delay, $\theta^{\prime}=10 \mathrm{~s}$.

Figure 3: The process in Example 1
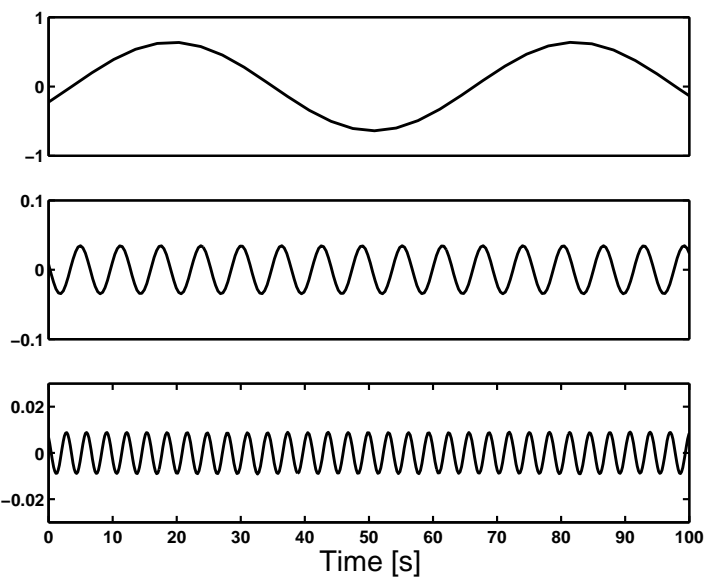

(a) No control
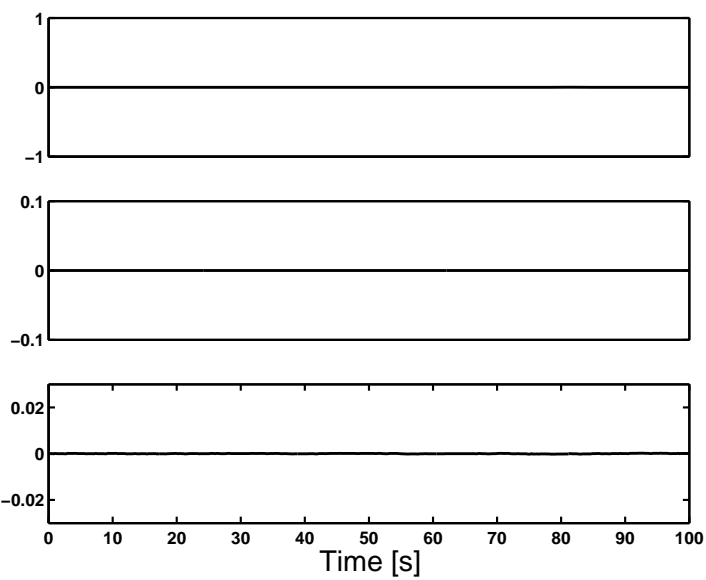

(b) Nominal case: Perfect control
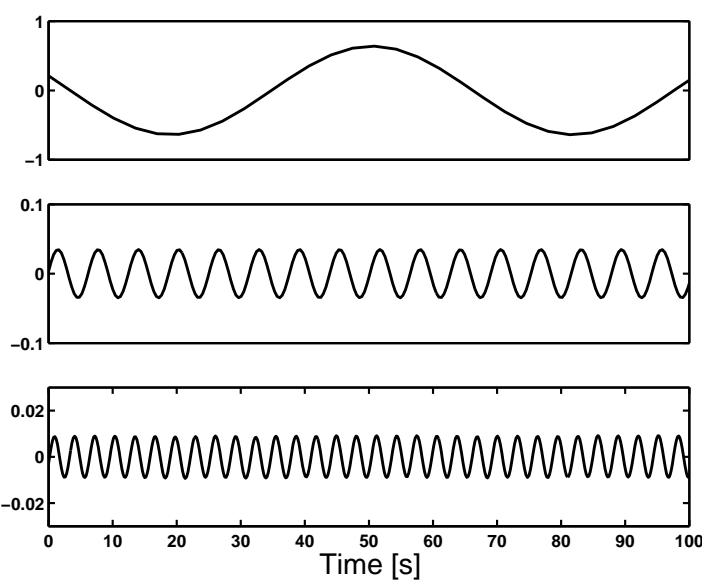

(c) Gain error $\left(k_{d}^{\prime}=0.5 k_{d}\right)$ : Same amplitude as with no control (for all frequencies) 

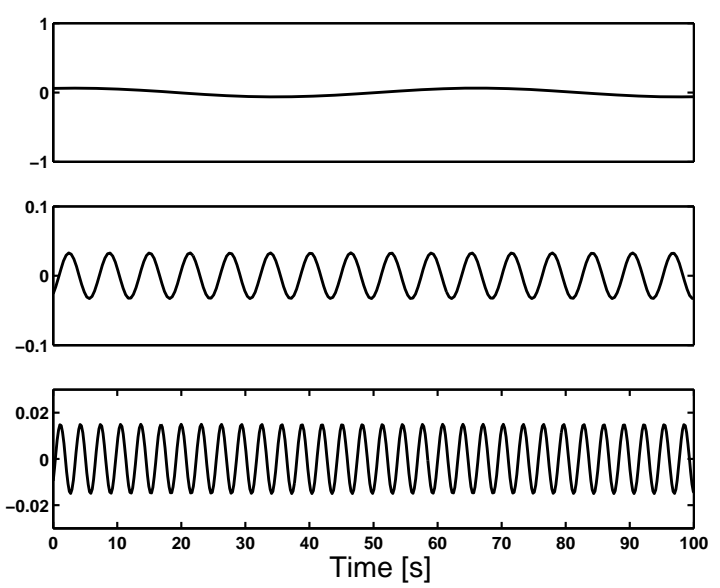

(d) Delay error $(|\Delta \theta|=1)$ : Improved performance for $0.1 \mathrm{rad} / \mathrm{s}$, no effect for $1 \mathrm{rad} / \mathrm{s}$, and larger amplitude for $2 \mathrm{rad} / \mathrm{s}$.
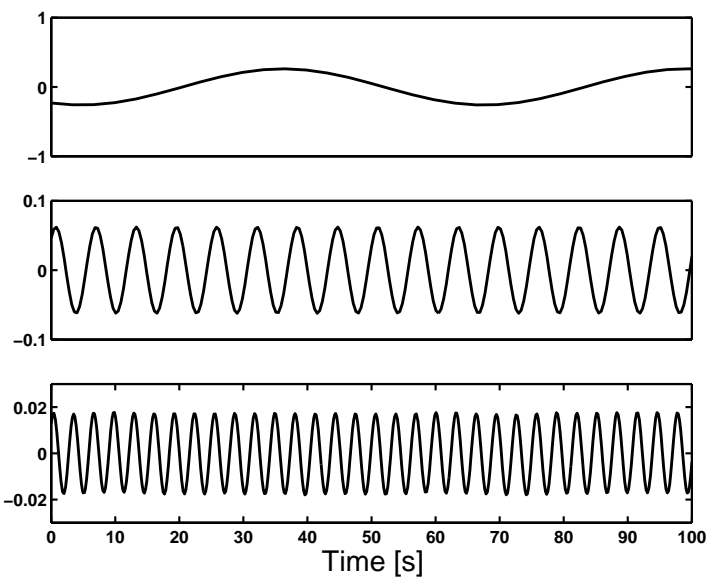

(e) Error in time constant $\left(\tau_{1}^{\prime}=3 \tau_{1}\right)$ : Improved performance only for the lowest frequency.
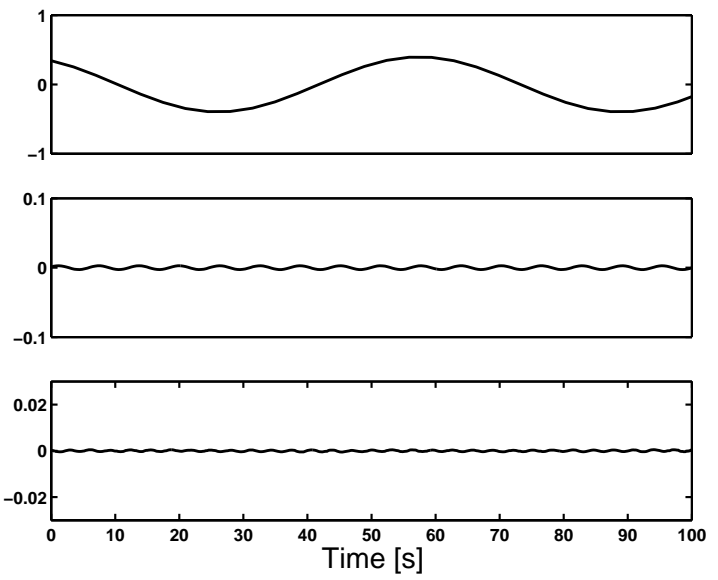

(f) Error in gain and time constant $\left(k_{d}^{\prime}=0.5 k_{d}\right.$ and $\left.\tau_{1}^{\prime}=0.5 \tau_{1}\right)$ : Improved performance for the two highest frequencies.

Figure 4: Feedforward control of two tank process: Response $\left(y=T_{2}\right)$ to sinusoidal disturbances $\left(d=T_{i n}=\sin \omega t\right.$ with frequencies $0.1,1$ and $2 \mathrm{rad} / \mathrm{s}$ (upper, middle and lower plot, respectively) nominal delay of $10 \mathrm{~s}$ is included manually in the feedforward controller.

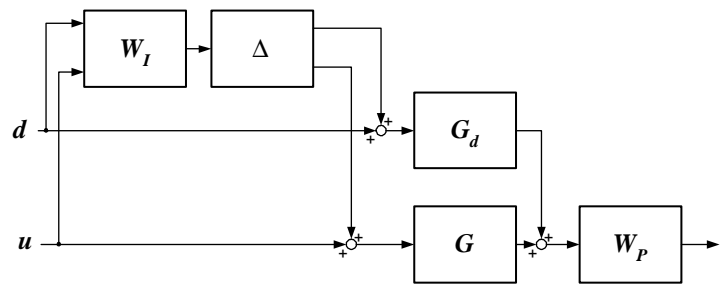

Figure 5: Problem formulation for the design of a $\mu$-optimal feedforward controller

The resulting $\left|S_{\mathrm{ff}}\right|$ is seen in Figure 6. From Figure 6(b) we see that "detuning" $\left(W_{P}<\infty\right)$ gives little improvement when there is a delay error, except when very large detuning $\left(W_{P} \leq\right.$ 1) is used.

With a low weight on performance relative to the uncertainty (small $W_{P}$ ), the $\mu$-optimal feedforward controller approaches no control $\left(\left|S_{\mathrm{ff}}\right|=1 ; \forall \omega\right)$. Interestingly, with large weight on performance (large $\left.W_{P}\right)$ we obtain a feedforward controller close to the ideal.

\section{Conclusions}

In this paper we have defined the feedforward "sensitivity functions", $S_{\mathrm{ff}}^{\prime}$ and $S_{\mathrm{ff}, r}^{\prime}$ for the disturbance and the reference, respectively. For ideal feedforward controllers, $K_{\mathrm{ff}}^{*}=G^{-1} G_{d}$ and $K_{\mathrm{ff}, r}^{*}=G^{-1}$ we find that $S_{\mathrm{ff}}^{\prime *}$ is equal to the relative error in $G / G_{d}$, and $S_{\mathrm{ff}, r}^{\prime *}$ is equal to the relative error in $G$ (except for the signs). A simple frequency domain analysis of $\left|S_{\mathrm{ff}}\right|$ and $\left|S_{\mathrm{ff}, r}\right|$ shows for which frequencies feedforward control has a positive (dampening) effect when certain uncertainties are present (in gain, delay, dominant time constant and a common combination of gain and time constant). The results are summarized in Figure 2.

The ideas are illustrated with a process example.

\section{Acknowledgements}

Financial support from the Research Council of Norway (NFR) and the first author's previous employer Norsk Hydro ASA is gratefully acknowledged. The authors also wish to thank Ass. Prof. Torsten Wik, Chalmers University of Techology, Sweden, for useful comments.

\section{References}

[1] J. G. Balchen. Reguleringsteknikk Bind 1 (In Norweigan) 1. Ed. Tapir, Trondheim, Norway, 1968.

[2] J. G. Balchen and K. I. Mummé. Process Control. Structures and Applications. Van Nostrand Reinhold, New York, 1988. 


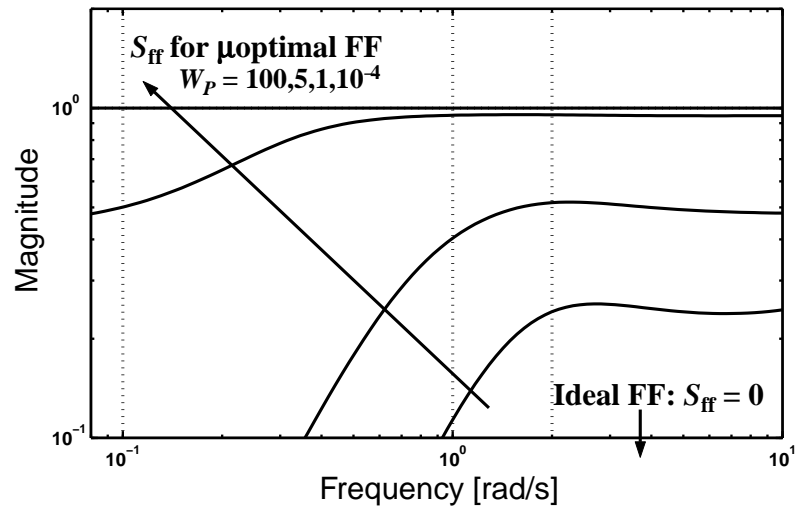

(a) Nominal case (no uncertainty)

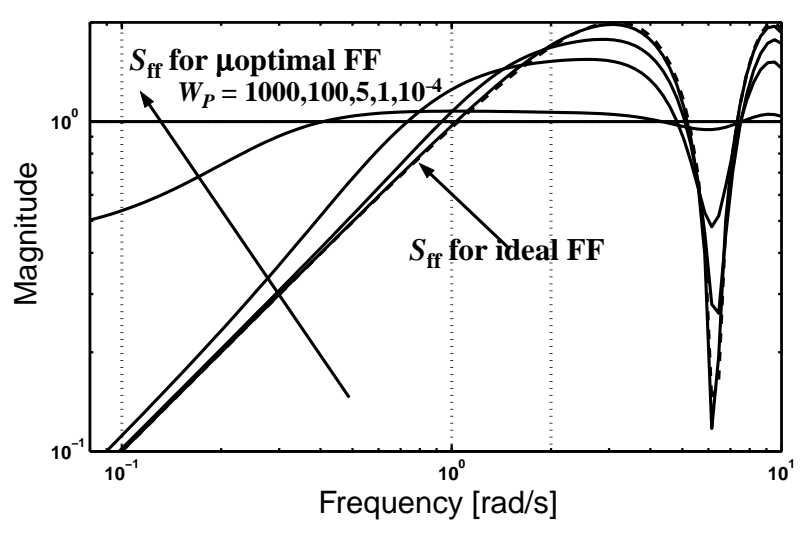

(b) Delay error $\left(\theta_{d}^{\prime}=\theta_{d}-1 \mathrm{~s}\right)$

Figure 6: Effect of detuned feedforward control: $\left|S_{\mathrm{ff}}\right|$ for $\mu$-optimal feedforward controllers with performance weight, $W_{P}=1000,100,5,1,10^{-4}$. ( $\left|S_{\mathrm{ff}}\right|$ for the "ideal" controller is dashed.)
[3] P. S. Buckley. Techniques of Process Control. John Wiley \& sons, inc., New York, 1964.

[4] D. R. Coughanowr. Process Systems Analysis and Control. McGraw-Hill Inc., New York, 1991.

[5] E. O. Doebelin. Control System Principles and Design. John Wiley \& Sons, New York, 1985.

[6] P. Harriott. Process Control. McGraw-Hill, New York, 1964.

[7] D. R. Lewin and C. Scali. Feedforward control in presence of uncertainty. Ind. Eng. Chem. Res., pages 23232331, 1988.

[8] T. E. Marlin. Process Control. Designing Processes and Control Systems for Dynamic Performance. McGrawHill, Inc., New York, 1995.

[9] R. H. Middleton and G. C. Goodwin. Digital Control and Estimation. Prentice-Hall International, London, 1990.

[10] M. Morari and E. Zafiriou. Robust Process Control. Prentice Hall, Englewood Cliffs, N.J., USA, 1989.

[11] K. Ogata. Modern Control Engineering. Prentice-Hall, Inc., Englewood Cliffs, N.J., USA, 1996.

[12] C. Scali, M. Hvala, and D. R. Lewin. Robustness issues in feedforward control. ACC-89, pages 577-581, 1989.

[13] D. Seborg, T. F. Edgar, and D. A. Mellichamp. Process Dynamics and Control. John Wiley \& Sons, New York, 1989.

[14] F. G. Shinskey. Process Control Systems - Application, Design, and Tuning 4th Ed. McGraw-Hill Inc., New York, USA, 1996.

[15] S. Skogestad and I. Postlethwaite. Multivariable Feedback Control. John Wiley \& Sons, Chichester, New York, 1996.

[16] G. Stephanopoulos. Chemical Process Control: An Introduction to Therory and Practice. Prentice-Hall International, Englewood Cliffs, New Jersey, USA, 1984. 